\title{
Opportunities for XML Data Mining in Modern Applications, or XML Data Mining: Where Is the Ore?
}

\author{
Stephane Bressan, Anthony Tung, and Yang Rui \\ Department of Computer Science, School of Computing, \\ National University of Singapore \\ steph@nus.edu.sg
}

\begin{abstract}
We attempt to identify the opportunities for XML data mining in modern applications. We will try and match requirements of modern application managing XML data with the capabilities of the existing XML mining tools and techniques.
\end{abstract}

